\title{
BMJ Global Health Simulating the impact of excise taxation for disease prevention in low-income and middle-income countries: an application to South Africa
}

\author{
Nicholas Stacey, ${ }^{1}$ Amit Summan, ${ }^{2}$ Aviva Tugendhaft, ${ }^{1}$ Ramanan Laxminarayan, ${ }^{2}$ \\ Karen Hofman ${ }^{1}$
}

To cite: Stacey N, Summan A, Tugendhaft A, et al. Simulating the impact of excise taxation for disease prevention in lowincome and middle-income countries: an application to South Africa. BMJ Glob Health 2018:3:e000568. doi:10.1136/ bmjgh-2017-000568

Handling editor Seye Abimbola Received 12 September 2017 Revised 12 November 2017 Accepted 13 December 2017

\section{(a) CrossMark}

${ }^{1}$ Priority Cost Effective Lessons for Systems Strengthening, School of Public Health, Faculty of Health Sciences, University of the Witwatersrand, Johannesburg, South Africa ${ }^{2}$ Center for Disease Dynamics Economics and Policy, Washington, District of Columbia, USA

Correspondence to Nicholas Stacey; nick.stacey@wits.ac.za

\section{ABSTRACT}

Introduction Excise taxes are policy tools that have been applied internationally with some success to reduce consumption of products adversely impacting population health including tobacco, alcohol and increasingly junk foods and sugary beverages. As in other low-income and middle-income countries, South Africa faces a growing burden of lifestyle diseases; accordingly we simulate the impact of multiple excise tax interventions in this setting. Methods We construct a mathematical model to simulate the health and revenue effects of increased excise taxes, which is adaptable to a variety of settings given its limited data requirements. Applying the model to South Africa, we simulate the impact of increased tax rates on tobacco and beer and of the introduction of a tax on sugar-sweetened beverages (SSB). Drawing on surveys of product usage and risk factor prevalence, the model uses a potential impact fraction to simulate the health effects of tax interventions. Results Adopting an excise rate of $60 \%$ on tobacco would result in a gain of 858923 life-years $(95 \%$ uncertainty interval (UI) 480188 to 1310329 ), while adopting an excise rate of $25 \%$ on beer would result in a gain of 568 063 life-years (95\% UI 412110 to 775560$)$ and the adoption of a $20 \%$ tax on SSBs would result in a gain of 688719 life-years (95\% UI 321788 to 1079653 ).

Conclusion More aggressive excise tax policies on tobacco, beer and SSBs in South Africa could result in meaningful improvements in population health and raised revenue.

\section{INTRODUCTION}

Non-communicable chronic diseases (NCDs) are emerging as a significant cause of morbidity and mortality in South Africa. ${ }^{12}$ The country, however, faces a unique epidemiological transition trajectory, with a quadruple burden of disease that includes chronic infectious diseases, in the form of HIV/ AIDS and TB, compounded by high rates of maternal and child mortality and violence and injury.

Poor recent macroeconomic performance, in particular stagnant economic growth and a

\section{Key questions}

What is already known about this topic?

- The literature is dominated by studies of tobacco taxation with much focus on retrospective evaluation of tobacco policy in South Africa.

- These studies find that recent tobacco taxation has seen increases in price and a fall in smoking prevalence.

- Only one study attempts to simulate the health impact of tobacco taxation, while none exist for alcohol taxation. Some more recent studies simulate the impact of sugar-sweetened beverage (SSB) taxation focusing on specific disease outcomes.

What are the new findings?

- This study departs from the literature by prospectively evaluating the potential impact of multiple tax interventions using a consistent modelling approach.

- This study provides the first attempt to synthesise existing evidence to simulate the impact of excise tax policies across products in South Africa and to report results using a comparable population health outcome measure.

\section{Recommendations for policy}

- In conjunction with the existing literature, our results suggest that while South Africa has seen some success with excise taxation, there are significant population health gains to be made with higher tax rates on tobacco and beer and with the introduction of tax on SSBs.

- Revenue raised from these interventions while small relative to aggregate public receipts could be targeted to further amplify disease treatment or prevention efforts.

- The arguments made by vested interests against these policy changes need to be evaluated relative to the potential benefits this study has identified.

relative fall in corporate income tax revenue has resulted from the global financial crisis. The consequent re-emergence of a structural deficit has placed additional constraints on 
the extent to which public resources can be allocated to manage these intersecting epidemics. Moreover, the structure of the South African health system mirrors its economic inequality with high-quality expensive healthcare provided to approximately $15 \%$ of the population covered by private health insurance while the remaining $85 \%$ depend on an overburdened and under-resourced public healthcare system. ${ }^{13}$

South Africa aims to deliver universal health coverage by 2025 through a National Health Insurance Scheme (NHI) but its successful implementation will rely on addressing the contextual constraints described above. Cost-effective prevention of non-communicable disease must use population-level policy tools targeting social determinants and not simply costly individual-level health service interventions. ${ }^{4}$ These tools include fiscal instruments such as taxes and subsidies, which can influence the price and affordability of products related to risk factors. Ideally fiscal policies would be implemented alongside complementary regulatory measures related to advertising and informative product labelling. While taxation of tobacco and alcohol has been in place to varying degrees internationally and in South Africa, there is now growing global interest in the use of fiscal tools as a means to improve diet and to reduce diet-related non-communicable disease. ${ }^{5}$ The WHO has called for global action to curtail the impact the of sugary drink consumption including the levying of taxes on these beverages.

South Africa's experience with excise taxation is mixed. While the prevalence of smoking has fallen in South Africa, recent population survey estimates suggest that $18.2 \%$ of adults still identify as current smokers. Since the early 1990s, South Africa has committed to an aggressive tobacco taxation policy generally regarded as a public health success with both taxes and tobacco prices rising significantly and consumption and smoking prevalence falling. ${ }^{6}$ However, in recent years, tax increases have been muted with a concomitant slowing in progress on tobacco consumption reduction. With an effective excise benchmark rate of $40 \%$ of retail price of the most popular brand, South Africa ranks far below the Framework Convention on Tobacco Control and WHO's recommendation of excise taxes constituting $70 \%$ of the price of the most popular brand of cigarettes. ${ }^{7}$

Despite significant alcohol intake and alcohol-related harms stretching across South Africa's quadruple burden of disease, excise taxes on alcohol have lagged behind those on tobacco. Of consequence is the rate on beer, which disproportionately contributes to alcohol intake in the South African population. Excise rates on beer are set based on a $23 \%$ of retail price benchmark and unlike tobacco have not increased significantly, with only a ZAR 1.18 per litre real increase since 2002. The volume of beer sold has risen from 2812 to 3565 million litres in this period.

In recent years (2003-2012), obesity prevalence in South Africa has increased among men by $2 \%$, from $9 \%$ to $11 \%$, and among women by $12 \%$, from $27 \%$ to $39 \%$, with a contemporaneous rise in sugar-sweetened beverage (SSB) intake. ${ }^{9} 10$ In response to rising obesity prevalence and diet-related disease, a tax on sugary beverages has been proposed but is yet to be legislated or implemented. ${ }^{11}$ Consumption of SSBs is closely linked to the onset of obesity and associated metabolic conditions. ${ }^{12-14}$

Collectively, the taxation of cigarettes, beer and SSBs target a significant source of the non-communicable disease burden. The Global Burden of Disease study attributes $4.96 \%$ of disability-adjusted life years (DALYs), $4.60 \%$ of DALYs and $5.98 \%$ of DALYS in South Africa to smoking, alcohol intake and high body mass-index, respectively. This study synthesises the available evidence on product use, risk factor prevalence, price responsiveness and mortality risk in a consistent mathematical modelling framework to produce estimates of the health and revenue gains of alternative excise tax policies on tobacco, alcohol and SSBs for South Africa. The findings suggest that in South Africa and other settings with similar constraints on public finances and healthcare resources, excise taxes could provide a means to simultaneously prevent disease, improve population health and raise revenue.

\section{METHODS \\ Modelling overview}

Building on the health impact assessment and modelling literature prospectively estimating the impacts of population-level interventions, this study provides estimates of the impact of tobacco, alcohol and SSB tax policy scenarios on population health outcomes in South Africa over a prospective 30 -year period. ${ }^{15-18}$ The approach incorporates economic simulation of consumption changes in response to tax-induced price changes and epidemiological simulation of the change in mortality associated with reduced use of the taxed products. A detailed mathematical description of the modelling is provided in online supplementary appendix.

An overview of the model's structure is presented in figure 1 . The model begins by including a tax on the particular product and assuming the tax will be passed through to the retail price facing consumers. Existing evidence from South Africa and other low and middle-income countries (LMIC) suggests that excise taxes are entirely or indeed overshifted to consumers. ${ }^{19} 20$ To quantify the change in consumption resulting from the tax-induced price change, we use price elasticities. A price elasticity is a unitless measure of the proportionate change in the demand of a product for a proportionate change in its price. ${ }^{21-23}$ Using estimated tax-induced price changes and own-price and cross-price elasticities, we simulate reductions in consumption of the taxed product and potential substitute or compliment products (see Section 1.3 of the online supplementary appendix).

The simulated changes in consumption of the targeted products lead to shifts in the distribution of a related risk factor. Reductions in SSB consumption lead to 


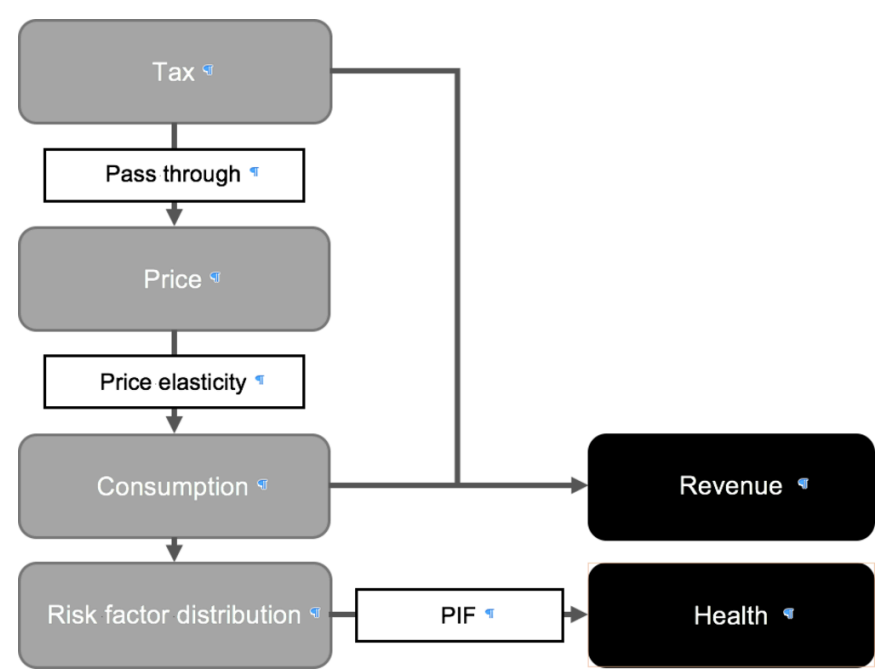

Figure 1 Model structure.

reductions in energy intake and in body-mass index, reductions in beer intake leads to reductions in absolute alcohol intake and reductions in cigarette consumption lead to changes in the prevalence of current smoking and former smoking. For beer and SSBs, we allow for substitution to other products (ie, beer to wine or spirits and SSBs to diet beverages, water and milk). We account for gender differences in product usage through the use of gender-specific baseline distributions of consumption and risk factors.

Drawing on estimates of all-cause mortality relative risks, we construct potential impact fractions (PIF) to adjust prevailing age-specific and gender-specific mortality rates, providing estimates of mortality in the presence of the tax scenario and its induced behaviour change (see Section 1.4 of the online supplementary appendix). A baseline population projection is constructed, assuming maintenance of the status quo. A simulation population projection is constructed, employing the PIF-adjusted age-specific and gender-specific mortality rates. The differences in mortality outcomes (deaths and life-years) between the baseline and simulation projections provide an estimate of the intervention effect. We discount the life-year outcomes assuming a discount rate of $3 \%$ (we report results assuming various discount rates in Section 3 of the online supplementary appendix). We do not incorporate fertility into these projections and thus the resulting impact is conservative and should be thought of as accruing only to the current South African population. Data sources are presented in table 1. Uncertainty arising from the statistical variation in input parameter estimates is addressed by Monte-Carlo simulation. We report the mean of the resulting distribution as the point-estimate of the outcomes of interest and the 2.5th percentile and 97.5 th percentiles as a $95 \%$ uncertainty interval (UI). The models were implemented in Microsoft Excel 2016 (with Visual Basic for Applications).
Table 1 Data sources

\begin{tabular}{|c|c|}
\hline Model input & Source \\
\hline \multicolumn{2}{|l|}{ General } \\
\hline \multicolumn{2}{|l|}{ Population } \\
\hline Age-sex structure & Statistics South Africa \\
\hline Age-specific mortality rates & IHME GBD 2015 \\
\hline \multicolumn{2}{|l|}{ Price and tax } \\
\hline Product prices & $\begin{array}{l}\text { Statistics South Africa, } \\
\text { National budget review }\end{array}$ \\
\hline Existing excise duties & National budget review \\
\hline \multicolumn{2}{|l|}{ Intervention-specific } \\
\hline \multicolumn{2}{|l|}{ Cigarettes } \\
\hline Price elasticity & IARC \\
\hline $\begin{array}{l}\text { Smoking prevalence and } \\
\text { intensity }\end{array}$ & $\begin{array}{l}\text { National income dynamics } \\
\text { study }\end{array}$ \\
\hline Mortality relative risk & Gettert et al (2012) \\
\hline \multicolumn{2}{|l|}{ Beer } \\
\hline Price elasticity & Authors' calculations \\
\hline Alcoholic beverage intake & $\begin{array}{l}\text { All media and products } \\
\text { survey }\end{array}$ \\
\hline Mortality relative risk & Di Castelnouvo et al (2006) \\
\hline \multicolumn{2}{|l|}{ SSB } \\
\hline Price elasticity & $\begin{array}{l}\text { Cabrera-Escobar et al } \\
(2013)\end{array}$ \\
\hline Beverage intake & $\begin{array}{l}\text { All media and products } \\
\text { survey }\end{array}$ \\
\hline Age-sex BMI distributions & $\begin{array}{l}\text { National income dynamics } \\
\text { study }\end{array}$ \\
\hline Mortality relative risk & Freedman et al (2006) \\
\hline
\end{tabular}

Details on parameter values and further mathematical detail are provided in online supplementary appendix.

BMI, body mass index; SSB, sugar-sweetened beverage.

\section{Scenarios modelled}

For each of the taxation interventions studied, we simulate the health benefits for three different tax rates: low, medium and high. The particular interventions as well as scenario rates were identified through a series of consultative meetings convened with policy-makers and experts in the South African policy environment and with the goal of guiding study of the potential for fiscal policy interventions to improve health. The scenarios modelled are presented in table 2. For each product, the rates for the various scenarios correspond to a tax burden relative to retail price expressed in percentage terms. Such percentage of retail price benchmarks is used for setting excise rates in South Africa and elsewhere. Various products would fall under the banners of tobacco, alcohol or SSBs. As such, we focus our modelling on the particular products highlighted in table 2. While all forms of tobacco are taxed, the most common form of tobacco consumption is cigarette smoking. ${ }^{24}$ We focus on beer as the largest source of absolute alcohol intake among 


\begin{tabular}{|c|c|c|c|c|}
\hline \multirow[b]{2}{*}{ Product } & \multirow[b]{2}{*}{ Baseline } & \multicolumn{3}{|c|}{ Intervention scenario } \\
\hline & & Low & Medium & High \\
\hline Cigarettes & $40 \%$ & $50 \%$ & $60 \%$ & $70 \%$ \\
\hline $\begin{array}{l}\text { Cigarettes (ZAR per } \\
\text { 20) }\end{array}$ & 12.42 & 19.64 & 32.07 & 58.52 \\
\hline Beer & $23 \%$ & $25 \%$ & $27 \%$ & $29 \%$ \\
\hline $\begin{array}{l}\text { Beer (ZAR per litre } \\
\text { AA) }\end{array}$ & 73.05 & 80.36 & 91.31 & 102.27 \\
\hline $\begin{array}{l}\text { Sugar-sweetened } \\
\text { beverages }\end{array}$ & $0 \%$ & $10 \%$ & $20 \%$ & $30 \%$ \\
\hline $\begin{array}{l}\text { Non-diet } \\
\text { carbonated drinks } \\
\text { (ZAR per litre) }\end{array}$ & 00.00 & 0.78 & 1.56 & 2.34 \\
\hline
\end{tabular}

Baseline rates taken from the national budget review. $\mathrm{AA}$, absolute alcohol.

alcoholic beverages and on non-diet carbonated soft drinks as the largest source of sugar intake across soft drink types. ${ }^{10}$

\section{RESULTS}

The interventions modelled find significant gains in years of life lived through reductions in premature mortality, as reported in table 3 . For cigarettes, the adoption of the medium scenario, a $60 \%$ rate, would result in a gain of 858923 life-years (95\% UI 480188 to 1310 329). For beer, the adoption of the medium scenario, a $27 \%$ rate, would result in a gain of 568063 life-years (95\% UI 412 110 to 775560$)$. And for SSBs the medium scenario, a $20 \%$ rate, would result in a gain of 688719 life-years over 30 years (95\% UI 321788 to 1079653 ). Across the three interventions the health effects are increasing in the rate adopted. For instance, for the SSB low scenario the gain in life-years rises from 340408 (95\% UI 144787 to 526 862 ) to 953158 (95\% UI 432661 to 1547834 ) in the high scenario. This result arises from the assumption of a constant elasticity and a greater tax inducing a greater price increase.

The use of the targeted products is more prevalent among men than women. ${ }^{152425}$ For the tobacco and alcohol interventions, this is reflected in the resulting health gains. For example, under the tobacco medium scenario, the gain in life-years is 605807 (95\% UI 324 376 to 948893 ) among men, but only 253116 (95\% UI 143604 to 373964 ) among women. The exception to this pattern occurs with SSBs, where the gain in life years is approximately equal across males and females. This likely arises as while SSB intake is greater among men, body mass index is generally greater among women. ${ }^{26}$

The modelling suggests that non-trivial revenues could be raised from the interventions studied. We report estimates of changes in annual excise revenue from the taxation scenarios in table 4 . In the case of cigarettes, the gains in revenue decrease from 12897 million ZAR (95\% UI 8023 to 17761 ) under the medium scenario to 7628 million ZAR (95\% UI -14 548 to 28423 ) under the high scenario. This arising from gains in per unit revenue being offset by falling consumption.

\section{DISCUSSION}

The public discourse in South Africa on excise taxation is generally limited to the potential costs and political barriers to adopting such policies as is the case in other global settings. This paper provides evidence on the potential benefits of adopting various excise tax interventions against which the costs of these policies should be evaluated. The modelled shifts in behaviour induced by the tax interventions studied would avert deaths and

Table 3 Cumulative life-years gained across modelled interventions after 30 years

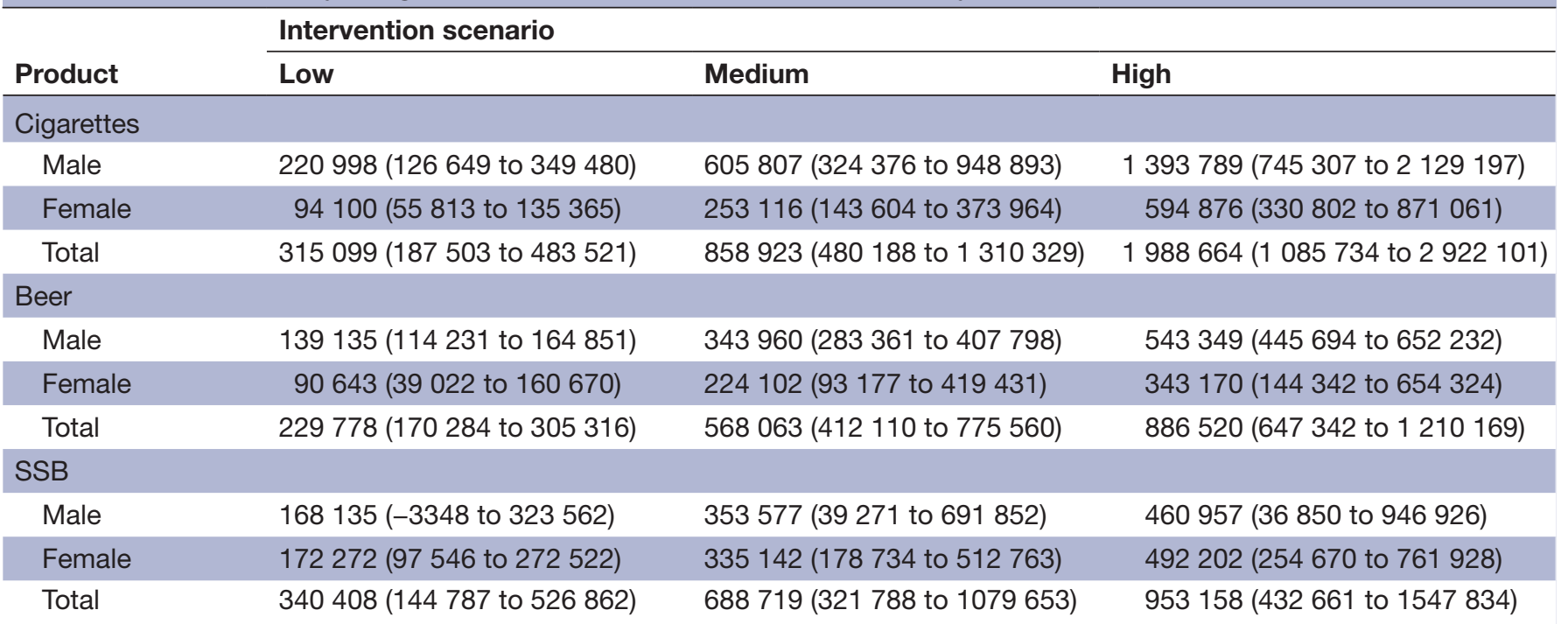

Uncertainty interval in brackets. We assume a discount rate of $3 \%$.

SSB, sugar-sweetened beverage. 
Table 4 Increases in annual revenues (Million ZAR)

\begin{tabular}{|c|c|c|c|}
\hline \multirow[b]{2}{*}{ Product } & \multicolumn{3}{|l|}{ Intervention scenario } \\
\hline & Low & Medium & High \\
\hline Cigarettes & 6278 (5162 to 7461$)$ & 12897 (8023 to 17761$)$ & $7628(-14548$ to 28423$)$ \\
\hline Beer & 11367 (11356 to 11378$)$ & 12916 (12 888 to 12947$)$ & 14315 (14 266 to 14363$)$ \\
\hline SSB & 4110 (3814 to 4413$)$ & $6513(5736$ to 7327$)$ & 7098 (5398 to 8474$)$ \\
\hline
\end{tabular}

SSB, sugar-sweetened beverage.

produce gains in life-years in a meaningful fashion and at the same time raise revenues. These shifts would have a significant impact on South Africa's growing NCD burden and would alleviate some of the strain on the healthcare system while simultaneously directing resources to other health needs.

The strengths of this study lie in the adoption of a simple but demonstrative modelling framework that is populated with local data on product usage, price responsiveness and risk factor prevalence. The results provide the first modelling evidence of alcohol taxation interventions in South Africa and contribute to a growing literature on tobacco and SSB taxation in South Africa. The framework allows the estimation of health impacts of alternative tax-product interventions through a consistent mathematical structure and is transferable to other LMIC settings through its limited data requirements. The model only requires data on product usage and prevailing mortality rates which may be aggregated or disaggregated by age and gender based on availability. Product usage is available in many LMIC settings through Demographic and Health Surveys, dietary and tobacco use surveys. Mortality data are available through the Global Burden of Disease Study. As consumption of harmful products in the high-income countries slows, corporations are shifting their focus to the low-income and middle-income countries. There is thus a significant need for analytical methods suitable for those settings that inform the adoption of appropriate disease prevention policies.

The analytical approach is subject to some weaknesses. Due to a lack of availability of disease prevalence and incidence for some diseases linked to the taxed products, we focus solely on mortality outcomes rather than a combination of morbidity and mortality. We thus underestimate the health benefit of the interventions considered and this could be meaningful considering that the illnesses averted are chronic and are potentially characterised by years lived in states of disability. Further, while we consider interventions on three separate products, we do not jointly model the impact of combinations of taxes on different products and therefore the resulting effects should be interpreted separately.

Comparisons to the international literature are not always meaningful due to differences in modelling methodology, differences in chosen outcome measure and contextual differences in setting characteristics like population size or prevailing population health. However, consistent with other studies, we find that these interventions could potentially induce significant population health gains. ${ }^{27-29}$

While a SSB tax remains to be implemented, existing alcohol and tobacco taxes would need to be strengthened in order to have greater impact. An often overlooked early success of postdemocracy South Africa was the adoption of an aggressive tobacco taxation policy which saw prices rise and smoking prevalence fall dramatically. In recent years, tax increases have slowed and with them slowing reductions in cigarette sales. A barrier to the pursuit of higher tobacco excise, often raised by industry, is the perceived threat of increased illicit trading of cigarettes. However, independent estimates of the size of the illicit market suggest it has remained relatively constant despite the earlier significant rises in tobacco taxes. ${ }^{30}$ Alcohol is linked to all of the components of South Africa's quadruple burden of disease, with beer serving as the largest source of alcohol intake and yet is subject to only moderate tax rates and increases. By increasing both alcohol and tobacco taxes to be more aligned with WHO standards and with the introduction of a SSB tax South Africa has the opportunity to effectively intervene before the point of healthcare delivery.

While we find uniformity in the scale of the potential health gains, we do identify heterogeneities across the tax interventions that are important for advocates and policy-makers to consider. In particular, gender differentials in the incidence of the health benefits of excise taxes vary across products. Much of the existing literature has considered the equity impacts of tax interventions by purely considering differential impacts along the income or wealth distribution. However, our study suggests gendered incidence of the benefits of excise taxes is significant and bears further consideration and research.

We find that while the health impacts are uniformly increasing in the rate adopted, this is not the case for revenues. For tobacco, we find that as rates rise, initially revenues increase but as consumption continues to fall revenues begin to fall as the per unit gains in revenue are offset by reduced overall product sales. This arises from the so-called Laffer curve, where revenue raised has a hump-shaped relationship to tax rate. That health is not subject to this phenomenon is important and has differential implications for policy-makers prioritising revenue raising as compared with policy-makers prioritising population health. This effect is only observed for tobacco due to the magnitude and range of rates modelled for 
the different products relative to their assumed price elasticities.

The relative magnitudes of revenues raised by the interventions considered is small relative to general annual revenue raising in South Africa of over one trillion Rand. Nevertheless, the revenues raised from the individual interventions range from $5 \%$ to $10 \%$ of annual public health expenditure. This revenue could be directed to the general revenue pool or could be directed to the prevention or treatment of the diseases caused by use of the particular products. These revenue gains would be complimented by cost-savings arising from reduced healthcare utilisation.

\section{CONCLUSION}

It is estimated that the proposed NHI scheme in South Africa will require expenditure of over R250 billion by 2025. The feasibility of the provision of universal health coverage under NHI in South Africa will rely in part on effective disease prevention interventions. In the broader context of South Africa's prevailing disease environment, constrained fiscal outlook and burdened healthcare system, fiscal interventions like excise taxes provide the opportunity for policy-makers to prevent disease, raise revenue and ease the burden of public healthcare facilities while attaining the goal of universal health coverage.

Acknowledgements The authors would like to thank the chairs and participants of the Inquiry on Fiscal Policies for Health process and reviewers of the resulting report which formed the basis for this work.

Contributors NS, AS, AT, RL and KH conceived the study. NS and AS constructed the mathematical model and ran the analyses. NS drafted the initial manuscript, while all coauthors reviewed, edited, and approved the final draft.

Funding The study was funded by the Bill and Melinda Gates Foundation (\#0PP1098574).

\section{Competing interests None declared.}

Provenance and peer review Not commissioned; externally peer reviewed.

Data sharing statement Model files and data available on request.

Open Access This is an Open Access article distributed in accordance with the terms of the Creative Commons Attribution (CC BY 4.0) license, which permits others to distribute, remix, adapt and build upon this work, for commercial use, provided the original work is properly cited. See: http://creativecommons.org/ licenses/by/4.0/

(C) Article author(s) (or their employer(s) unless otherwise stated in the text of the article) 2018. All rights reserved. No commercial use is permitted unless otherwise expressly granted.

\section{REFERENCES}

1. Mayosi BM, Flisher AJ, Lalloo UG, et al. The burden of non-communicable diseases in South Africa. The Lancet 2009;374:934-47.

2. Pillay-van Wyk V, Msemburi W, Laubscher R, et al. Mortality trends and differentials in South Africa from 1997 to 2012: second national burden of disease study. Lancet Glob Health 2016;4:e642-53.

3. Mayosi BM, Benatar SR. Health and health care in South Africa20 years after Mandela. N Engl J Med 2014;371:1344-53.
4. Cecchini M, Sassi F, Lauer JA, et al. Tackling of unhealthy diets, physical inactivity, and obesity: health effects and costeffectiveness. Lancet 2010;376:1775-84.

5. WHO Regional Office for Europe (Nutrition Physical Activity and Obesity Programme). Using price policies to promote healthier diets. Brussels: WHO European Regional Office, 2015:41.

6. van Walbeek C. Recent trends in smoking prevalence in South Africa-some evidence from AMPS data. S Afr Med $J$ 2002;92:468-72.

7. World Health Organisation. WHO technical manual on tobacco tax administration. Geneva: World Health Organisation Press, 2011.

8. Euromonitor International. 2015. http://www.euromonitor.com/

9. Shisana O, Labadarios D, Rehle T, et al. South African National Health and Nutrition Examination Survey (SANHANES-I). Cape Town: HSRC, 2013.

10. Euromonitor International. Soft drinks in South Africa. 2015.

11. National Treasury. Taxation of sugar-sweetened beverages. Pretoria: National Treasury, 2016

12. Malik VS, Popkin BM, Bray GA, et al. Sugar-sweetened beverages and risk of metabolic syndrome and type 2 diabetes: a metaanalysis. Diabetes Care 2010;33:2477-83.

13. Malik VS, Popkin BM, Bray GA, et al. Sugar-sweetened beverages, obesity, type 2 diabetes mellitus, and cardiovascular disease risk. Circulation 2010;121:1356-64.

14. Popkin BM, Hawkes C. Sweetening of the global diet, particularly beverages: patterns, trends, and policy responses. Lancet Diabetes Endocrinol 2016;4:174-86.

15. Manyema M, Veerman LJ, Chola L, et al. The potential impact of a $20 \%$ tax on sugar-sweetened beverages on obesity in South African adults: a mathematical model. PLoS One 2014;9:e105287.

16. Verguet S, Gauvreau CL, Mishra S, et al. The consequences of tobacco tax on household health and finances in rich and poor smokers in China: an extended cost-effectiveness analysis. Lancet Glob Health 2015;3:e206-216.

17. Brennan A, Meier P, Purshouse R, et al. The sheffield alcohol policy model - a mathematical description. Health Econ 2015;24:1368-88.

18. Briggs AD, Mytton OT, Kehlbacher A, et al. Overall and income specific effect on prevalence of overweight and obesity of $20 \%$ sugar sweetened drink tax in UK: econometric and comparative risk assessment modelling study. BMJ 2013;347:f6189.

19. Colchero MA, Salgado JC, Unar-Munguía M, et al. Changes in prices after an excise tax to sweetened sugar beverages was implemented in Mexico: evidence from urban areas. PLoS One 2015;10:e0144408.

20. Russell $C$, van Walbeek $C$. How does a change in the excise tax on beer impact beer retail prices in South Africa? South African Journal of Economics 2016;84:555-73.

21. Nghiem N, Wilson N, Genc M, et al. Understanding price elasticities to inform public health research and intervention studies: key issues. Am J Public Health 2013;103:1954-61.

22. Deaton A, Muellbauer J. Economics and consumer behavior. Cambridge, New York: Cambridge University Press, 1980.

23. Stacey N, Tugendhaft A, Hofman K. Sugary beverage taxation in South Africa: household expenditure, demand system elasticities, and policy implications. Prev Med 2017;105S:S26-31.

24. Groenewald P, Vos T, Norman R, et al. Estimating the burden of disease attributable to smoking in South Africa in 2000. S Afr Med J 2007;97(Pt 2):674-81.

25. Schneider M, Norman R, Parry C, et al. Estimating the burden of disease attributable to alcohol use in South Africa in 2000. S Afr Med J 2007;97(Pt 2):664-72.

26. Averett SL, Stacey N, Wang Y. Decomposing race and gender differences in underweight and obesity in South Africa. Econ Hum Biol 2014; 15:23-40.

27. Powell LM, Chriqui JF, Khan T, et al. Assessing the potential effectiveness of food and beverage taxes and subsidies for improving public health: a systematic review of prices, demand and body weight outcomes. Obes Rev 2013:14:110-28.

28. Holm AL, Veerman L, Cobiac L, et al. Cost-effectiveness of changes in alcohol taxation in Denmark: a modelling study. Cost Eff Resour Alloc 2014;12:1

29. Veerman JL, Sacks G, Antonopoulos N, et al. The impact of a tax on sugar-sweetened beverages on health and health care costs: a modelling study. PLoS One 2016;11:e0151460.

30. Blecher E. A mountain or a molehill: is the illicit trade in cigarettes undermining tobacco control policy in South Africa? Trends Organ Crime 2010;13:299-315 